\title{
Stainless Steel 304 Micro-pillar Mechanical Response to Ion Irradiation and Helium Implantation Under Transmission Electron Microscopy Observation
}

Ryan Schoell ${ }^{1}$, David Frazer ${ }^{2}$, Peter Hosemann ${ }^{3}$ and Djamel Kaoumi ${ }^{1}$

${ }^{1}$ North Carolina State University, Raleigh, North Carolina, United States, ${ }^{2}$ Los Alamos National Laboratory, Los Alamos, New Mexico, United States, ${ }^{3}$ University of California Berkeley, Berkeley, California, United States

Austenitic stainless steels make up various components inside light water nuclear reactors due to their desirable corrosion resistance, adequate mechanical properties, and radiation tolerance. Components like the bolts that are used to hold core internals are made from 304 Stainless Steels (SS) are subjected to the extreme environment near a nuclear core which includes elevated temperatures up to $350{ }^{\circ} \mathrm{C}$, mechanical stress, and neutron irradiation [1]. Additionally, helium originating from alpha decay and transmutation can become trapped in the material and alter its mechanical properties [2]. To better understand the role of irradiation damage and helium implantation on the mechanical response of 304SS, in situ micropillar compression tests were conducted. Other studies [3-5] perform some versions of hardness tests after irradiation and helium implantation and lose the ability to view the mechanical response of the irradiated material. In situ micropillar compression tests give the ability to view the evolution of the irradiated and helium implanted microstructure under stress under Transmission Electron Microscopy (TEM) observation. The use of micropillar compression also allows for the use of ions to simulate neutron irradiated with little ion implantation [6]. Micropillars were fabricated using focused ion beam techniques. To determine the geometry to use for micropillar compression testing, two kinds of pillars were created. One pillar geometry had a square cross section geometry with a width and thickness of $300 \mathrm{~nm}$ and a height of $500 \mathrm{~nm}$ (Thick Pillar). The other pillar was fabricated with a rectangular cross section geometry which was also had a width of $300 \mathrm{~nm}$ and a height of $500 \mathrm{~nm}$ but had a thickness of approximately 100 $\mathrm{nm}$ (Thin Pillar). The resultant stress-strain curves are evident in Figure 1. The thin pillar has many load drops compared to the thick pillar. The thin pillar was found to bend instead of compressed which can alter the results of the stress-strain curves. Although the thinner pillar is better for TEM imaging, it cannot provide reliable mechanical data. The large grain size of about 30-40 $\mu \mathrm{m}$ meant that each pillar was a single crystal and that groups of pillars had the same orientation.

\section{In Situ Ion Irradiation under TEM Observation}

Various experimental conditions were used to probe the mechanical properties of irradiated materials. Three pillars served as the control group and only underwent a $300{ }^{\circ} \mathrm{C}$ heat treatment for the duration of time the other pillars were irradiated at the same temperature. Two pillars were only irradiated with $1 \mathrm{MeV}$ Krypton ions at $300{ }^{\circ} \mathrm{C}$ until a dose of 5 dpa was reached at the Intermediate Voltage Electron Microscope (IVEM) facility at Argonne National Laboratory. Finally, other pillars were preimplanted with $25 \mathrm{keV}$ helium ions to a fluence of either $1 \times 10^{17} \mathrm{He}$ ion $/ \mathrm{cm} 2,5 \times 10^{17} \mathrm{He}$ ion $/ \mathrm{cm} 2$, or $1 \times 10^{18} \mathrm{He}$ ion $/ \mathrm{cm}^{2}$. The $\mathrm{Kr}$ ion irradiated only pillars showed the formation and disappearance of irradiation induced defects. The number density of defect clusters decreased while the size of the defect clusters increased with increasing dose. Before $\mathrm{Kr}$ irradiation, only the pillars implanted with $1 \times 10^{18} \mathrm{He}$ ion $/ \mathrm{cm}^{2}$ showed signs of cavities. After $\mathrm{Kr}$ irradiation, cavities could be seen in all pillars implanted with helium. The largest of cavities was seen in the pillars with the highest fluence of helium. 


\section{In Situ Micropillar Compression under TEM Observation}

Pillar compression tests were performed using a PI 95 Picoindenter from Hysitron $\bigodot$ which can accurately measure the force as a function of displacement. All pillar tests were conducted at a displacement rate of $7 \mathrm{~nm} / \mathrm{s}$. Load as a function of displacement curves were collected and converted to stress strain curves to extract mechanical properties. Due to the ambiguity of determining the yield stress from micropillar compression tests, the flow stress at 5\% strain was used. The flow stress of the $\mathrm{Kr}$ irradiated only pillar was larger than the flow stress of the control pillars at $5 \%$ strain. All the pillars implanted with helium followed by $\mathrm{Kr}$ irradiation had larger flow stresses than the $\mathrm{Kr}$ irradiated only pillars. Moreover, the flow stress decreased with increasing amounts of helium. It should be noted that the orientation of the different groups were slightly different with the $\mathrm{Kr}$ irradiated only pillars having a compression axis about $11^{\circ}$ from the [111] direction while the helium implanted pillars were about $21^{\circ}$ from the [111] direction. To quantify the amount of hardening that could be coming from the helium bubbles, the Friedel-Kroupa-Hirsch (FHK) hardening model was used [7]. The model under predicted the amount of hardening that should be seen when compared to the $\mathrm{Kr}$ ion irradiated only pillars. Specifically, the model predicts a hardening of $23.1 \mathrm{GPa}, 19.4 \mathrm{GPa}$, and $10.6 \mathrm{GPa}$ for the $1 \times 10^{17} \mathrm{He}$ ion $/ \mathrm{cm}^{2}, 5 \times 10^{17} \mathrm{He}$

ion $/ \mathrm{cm}^{2}$, and $1 \times 10^{18} \mathrm{He}$ ion/ $/ \mathrm{cm}^{2}$ pillars, respectively. Possible explanations for the deviation include that helium might not just be located inside the bubbles but might also be inside the crystalline lattice. Furthermore, the $25 \mathrm{keV}$ helium ions also lead to a considerable amount of damage in the first $150 \mathrm{~nm}$ of the pillars. These factors could have led to the additional hardening that the model does not predict.

Load drops in the stress strain curves were only evident in the pillars that were irradiated with $\mathrm{Kr}$ Ions, especially the pillars that were pre-implanted with helium. The presence of load drops in irradiated stress strain curves of micropillars has been found in other papers [8] and they are speculated to be associated with defect free channels. Snap shots of the $1 \times 10^{17} \mathrm{He}$ ion $/ \mathrm{cm}^{2}$ pillar over various strains with its accompanying stress strain curve is shown in Figure 2. 

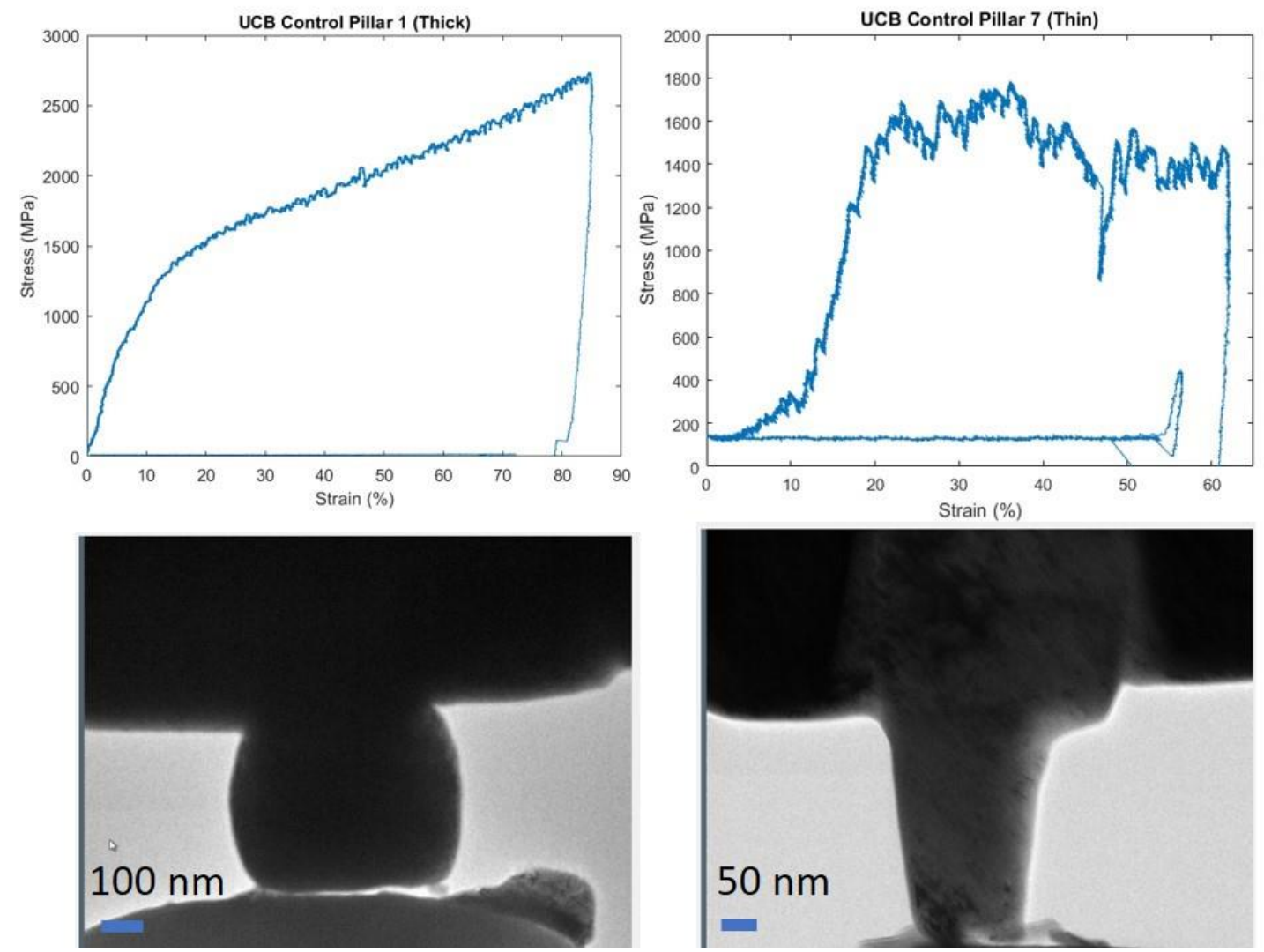

Figure 1. Stress-Strain Curves from a Thick (left) and Thin (Right) Pillar
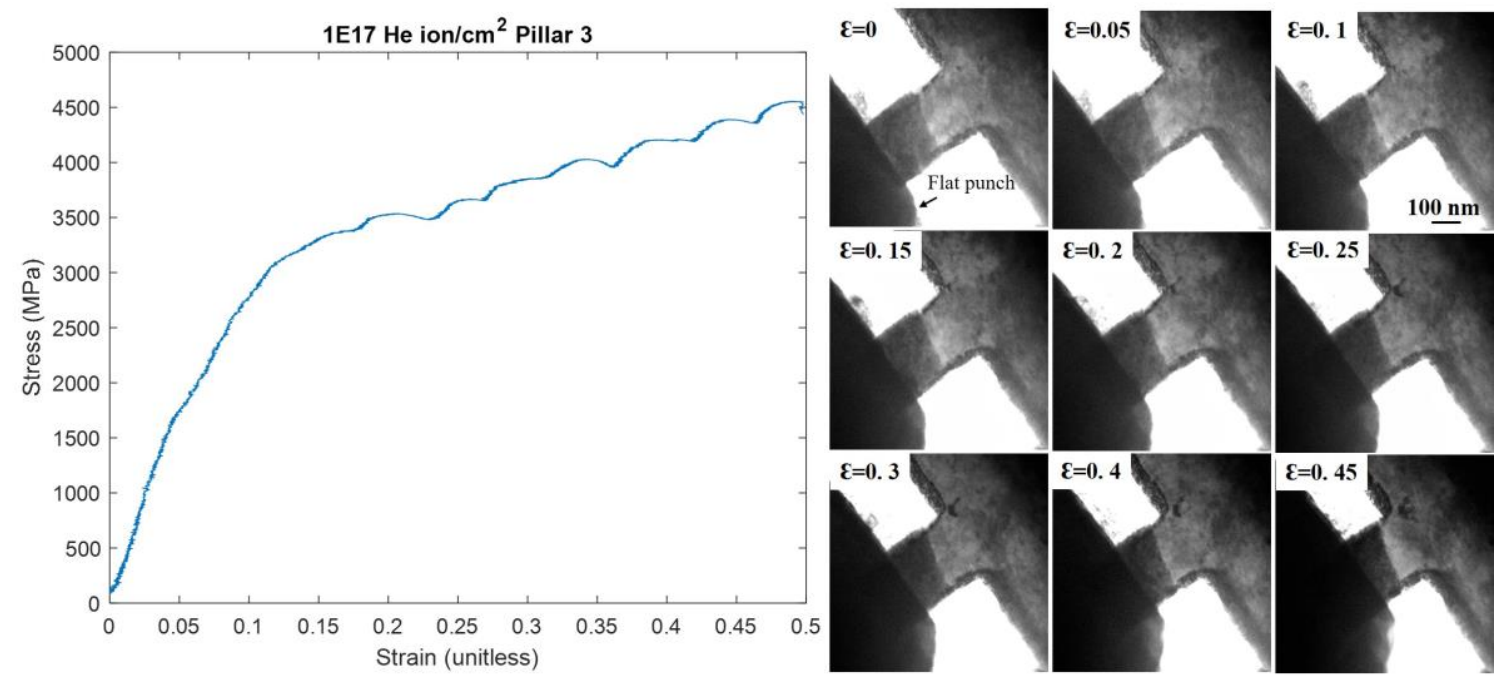

Figure 2. Snap shots of the $1 \times 1017 \mathrm{He}$ ion/cm2 pillar over Various Strains and the Stress Strain Curve

\section{References}

1. Neil E. Todreas, M.S.K., Nuclear Systems: Thermal Hydraulic Fundamentals. 2 ed, ed. C. Press. Vol. 1. 2011.

2. Villacampa, I., et al., Helium bubble evolution and hardening in $316 L$ by post-implantation annealing. Journal of Nuclear Materials, 2018. 500: p. 389-402. 
3. Heintze, C., et al., Irradiation hardening of Fe-9Cr-based alloys and ODS Eurofer: Effect of helium implantation and iron-ion irradiation at $300^{\circ} \mathrm{C}$ including sequence effects. Journal of Nuclear Materials, 2016. 470: p. 258-267.

4. Gao, J., K. Yabuuchi, and A. Kimura, Ion-irradiation hardening and microstructural evolution in F82H and ferritic alloys. Journal of Nuclear Materials, 2019. 515: p. 294-302.

5. Roldán, M., et al., Comparative study of helium effects on EU-ODS EUROFER and EUROFER97 by nanoindentation and TEM. Journal of Nuclear Materials, 2015. 460: p. 226-234.

6. Weaver, J.S., et al., Spherical nanoindentation of proton irradiated 304 stainless steel: A comparison of small scale mechanical test techniques for measuring irradiation hardening. Journal of Nuclear Materials, 2017. 493: p. 368-379.

7. Landau, P., et al., Deformation of as-fabricated and helium implanted 100nm-diameter iron nanopillars. Materials Science and Engineering: A, 2014. 612: p. 316-325.

8. Zhao, X., et al., In situ measurements of a homogeneous to heterogeneous transition in the plastic response of ion-irradiated 〈111〉 Ni microspecimens. Acta Materialia, 2015. 88: p. 121-135.

9. Ryan Schoell, D.Frazer, Ce Zheng, Peter Hosemann, Djamel Kaoumi, In Situ Micro-Pillar Compression Tests of 304 Stainless Steels After Ion Irradiation and Helium Implantation. Journal of The Minerals, Metals \& Materials, 2020. 